\title{
A non-perturbative effect of gluons for scalar diquark in the Schwinger-Dyson formalism
}

\author{
Shotaro Imai*, Hideo Suganuma \\ Department of Physics, Graduate School of Science, Kyoto University, \\ Kitashirakawa-oiwake, Sakyo, Kyoto 606-8502, Japan \\ E-mail: imaieruby.scphys.kyoto-u.ac.jp
}

The diquark has been considered to be important effective degree of freedom in hadron physics, especially for multi-quark physics and the structure of heavy hadronic states. Using the Schwinger-Dyson formalism, we investigate the non-perturbative effect of gluons for a scalar diquark with renormalization-group improved coupling in the Landau gauge. Here, we treat the scalar diquark as an effective degree of freedom with a peculiar size, while the diquark is originally a bound-state-like object of two quarks. Since the diquark has a non-zero color charge, it still strongly interacts with gluons. We evaluate the gluonic non-perturbative effect to the diquark, considering the size effect of the diquark. We investigate the mass function of the diquark in both cases with a constant bare diquark mass and twice of the running quark self-energy. It is found that the diquark, especially the small diquark, obtains a large effective mass by the gluonic dressing effect. The scalar diquark mass seems to be dynamically generated by the non-perturbative effect, although it does not have chiral symmetry explicitly.

XV International Conference on Hadron Spectroscopy-Hadron 2013

4-8 November 2013

Nara, Japan

${ }^{*}$ Speaker. 


\section{Introduction}

Recent experiments have discovered many heavy hadronic states including exotic states, which cannot be understood as ordinary hadrons [四]. In order to describe the structure of their states, the diquark is considered as an effective degree of freedom. For the diquark, the most attractive channel by the one-gluon exchange is the color and flavor anti-triplet $\overline{\mathbf{3}}_{c, f}$ and spin singlet with even parity $0^{+}$. If the diquark correlation is developed in a hadron such as a heavy baryon $(Q q q)$, this scalar diquark channel would be favored. The diquark is made by two quarks with gluonic interaction, and it still strongly interacts with gluons because of its non-zero color charge. The dynamics of diquark and gluons may affect the structure of a hadron.

In quark-hadron physics, the Schwinger-Dyson (SD) formalism is often used to evaluate the

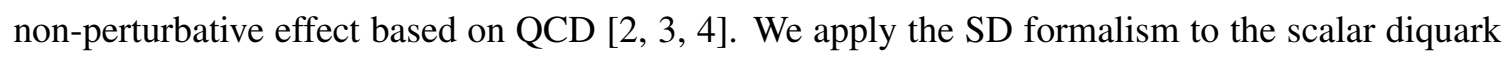
with its peculiar size, and investigate the effective diquark mass, which reflects a non-perturbative dressing effect by gluons. The diquarks are sometimes treated as point-like object or local boson fields. However, the diquark must have an effective size, since it is a bound-state-like object inside a hadron. Therefore, we investigate also the size effect of the diquark for its dynamics.

\section{The Schwinger-Dyson Equation for the Scalar Diquark}

We consider the scalar diquark as an effective degree of freedom with a peculiar size like a meson, assuming it to be an extended fundamental field $\phi(x)$ [目]. The scalar diquark interacts with gluons since it has non-zero color charge and is affected by non-perturbative gluonic effects [四].

To evaluate the non-perturbative effect, we take the Schwinger-Dyson (SD) formalism with the rainbow-ladder truncation with the Higashijima-Miransky approximation $\alpha_{S}\left(\left(p_{E}-k_{E}\right)^{2}\right) \approx$ $\alpha_{s}\left(\max \left(p_{E}^{2}, k_{E}^{2}\right)\right)$ in the Landau gauge. Here, $p_{E}$ denotes the Euclidean momentum. We use a renormalization-group(RG)-improved coupling in the case of $N_{c}=3$ and $N_{f}=3$,

$$
\alpha_{s}\left(p_{E}^{2}\right)=\frac{g^{2}\left(p_{E}^{2}\right)}{4 \pi}=\left\{\begin{array}{ll}
\frac{12 \pi}{11 N_{c}-2 N_{f}} \frac{1}{\ln \left(p_{E}^{2} / \Lambda_{\mathrm{QCD}}^{2}\right)} & \left(p_{E}^{2} \geq p_{\mathrm{IR}}^{2}\right) \\
\frac{12 \pi}{11 N_{c}-2 N_{f}} \frac{1}{\ln \left(p_{\mathrm{IR}}^{2} / \Lambda_{\mathrm{QCD}}^{2}\right)} & \left(p_{E}^{2} \leq p_{\mathrm{IR}}^{2}\right)
\end{array},\right.
$$

with an infrared regularization of a simple cut at $p_{\mathrm{IR}} \simeq 640 \mathrm{MeV}$ which leads to $\ln \left(p_{\mathrm{IR}}^{2} / \Lambda_{\mathrm{QCD}}^{2}\right)=$ $1 / 2$, and the QCD scale parameter $\Lambda_{\mathrm{QCD}}=500 \mathrm{MeV}$ [四, 团]. To include the size effect of diquark, we introduce a simple form factor in the 4D Euclidean space as

$$
f_{\Lambda}\left(p_{E}^{2}\right)=\left(\frac{\Lambda^{2}}{p_{E}^{2}+\Lambda^{2}}\right)^{2}
$$

where the momentum cutoff $\Lambda$ corresponds to the inverse of the diquark size $R$, and we here set $R \equiv \Lambda^{-1}$. The size effect of the diquark can be included in the vertex as $\alpha_{s}\left(p^{2}\right) \rightarrow \alpha_{s}\left(p^{2}\right) f_{\Lambda}\left(p^{2}\right)$.

The SD equation for the scalar diquark with the bare diquark mass $m_{\phi}$ is diagrammatically expressed as Fig. $⿴$ and is written by

$$
\begin{aligned}
\Sigma^{2}\left(p_{E}^{2}\right)= & m_{\phi}^{2}+\frac{8}{\pi} \int_{0}^{\infty} d k_{E} k_{E} \alpha_{s}\left(k_{E}^{2}\right) f_{\Lambda}\left(k_{E}^{2}\right) \\
& -\frac{2 \alpha_{s}\left(p_{E}^{2}\right) f_{\Lambda}\left(p_{E}^{2}\right)}{\pi p_{E}^{2}} \int_{0}^{p_{E}} d k_{E} \frac{k_{E}^{5}}{k_{E}^{2}+\Sigma^{2}\left(k_{E}^{2}\right)}-\frac{2}{\pi} \int_{p_{E}}^{\infty} d k_{E} \frac{\alpha_{s}\left(k^{2}\right) f_{\Lambda}\left(k_{E}^{2}\right) k_{E}}{k_{E}^{2}+\Sigma^{2}\left(k_{E}^{2}\right)} .
\end{aligned}
$$




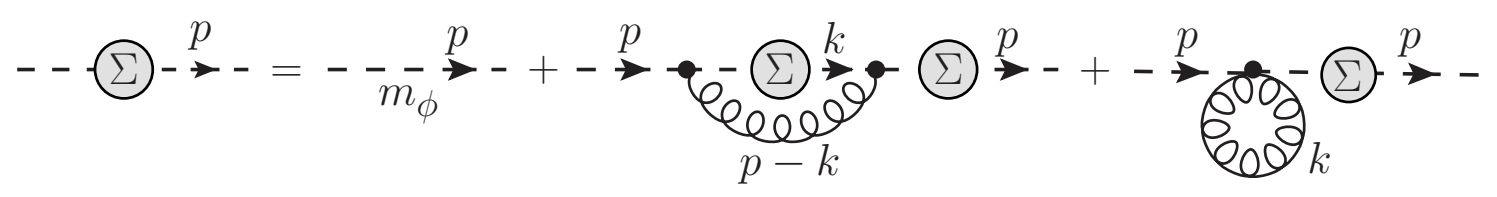

Figure 1: The Schwinger-Dyson equation for the scalar diquark. The shaded blob is the self-energy $\Sigma\left(p^{2}\right)$, the dashed line denotes the scalar diquark propagator and the curly line the gluon propagator. The last diagram in RHS is peculiar term of scalar theory.

\section{Numerical Result}

\subsection{The Parameters in the Diquark Theory}

The bare mass $m_{\phi}$ and the size $R$ (inverse of cutoff $\Lambda$ ) are free parameters of the diquark theory. Since the diquark is originally made of two quarks, the bare diquark mass can be simply considered as twice of the quark mass. In this paper, we consider two cases of the bare diquark mass. One is twice of the constituent quark mass, i.e., $m_{\phi}=600 \mathrm{MeV}$. The other is twice of the running quark self-energy, i.e., $m_{\phi}\left(p_{E}^{2}\right)=2 \Sigma_{q}\left(p_{E}^{2}\right)$, where $\Sigma_{q}\left(p_{E}^{2}\right)$ is determined by the SD equation for single

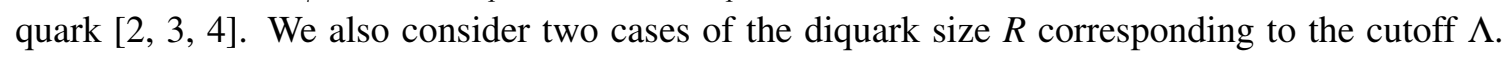
One is the typical size of a baryon $R=1 \mathrm{fm}$, i.e., $\Lambda=200 \mathrm{MeV}$, which gives the upper limit of the size (the lower limit of the cutoff). The second is the typical size of a constituent quark $R \simeq 0.3 \mathrm{fm}$, i.e., $\Lambda=600 \mathrm{MeV}$, which gives the lower limit of the size (the upper limit of the cutoff).

\subsection{The Constant Bare Mass Case}

We first show in Fig. $\square$ the case of the constant bare mass $m_{\phi}=600 \mathrm{MeV}$ with dependence on the diquark size $R$. The diquark self-energy $\Sigma\left(p_{E}^{2}\right)$ is always larger than the bare mass $m_{\phi}$ and almost constant except for a small bump structure at an infrared region. The value of the self-energy strongly depends on the size $R$, e.g., the "compact diquark" with $R \simeq 0.3 \mathrm{fm}$ has a large mass.

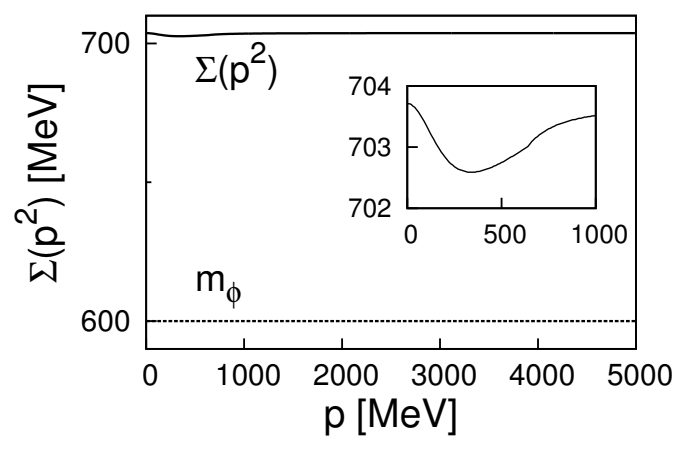

(a) $R=1 \mathrm{fm} \quad$ (i.e., $\Lambda=200 \mathrm{MeV}$ )

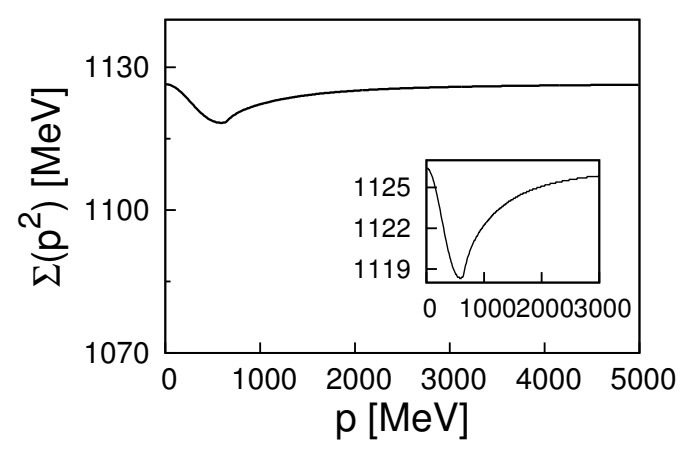

(b) $R \simeq 0.3 \mathrm{fm} \quad$ (i.e., $\Lambda=600 \mathrm{MeV}$ )

Figure 2: The scalar-diquark self-energy $\Sigma\left(p^{2}\right)$ as a function of the momentum in the constant bare-mass case of $m_{\phi}=600 \mathrm{MeV}$ with [a] $R=1 \mathrm{fm}$ and [b] $R \simeq 0.3 \mathrm{fm}$. In both cases, there appears a small bump structure, which is displayed in the small window. In the left figure, the original bare mass $m_{\phi}$ is plotted for comparison. 


\subsection{The Running Bare Mass Case}

We show in Fig. [1] the case of the running bare mass $m_{\phi}\left(p_{E}^{2}\right)=2 \Sigma_{q}\left(p_{E}^{2}\right)$ with dependence on $R$. The diquark self-energy $\Sigma\left(p_{E}^{2}\right)$ also strongly depends on the diquark size $R$. In the low-momentum region, the behavior of $\Sigma\left(p_{E}^{2}\right)$ reflects the running property of the bare mass, especially in the $R=1$ fm case, the gluonic effect seems to be small, because of $\Sigma\left(p_{E}^{2}\right) \approx 2 \Sigma_{q}\left(p_{E}^{2}\right)$. In the high-momentum region, the diquark self-energy keeps a large finite value, while the bare mass $m_{\phi}\left(p_{E}^{2}\right)$ goes to zero. This suggests the mass generation of the scalar diquark by the gluonic radiative correction [6].

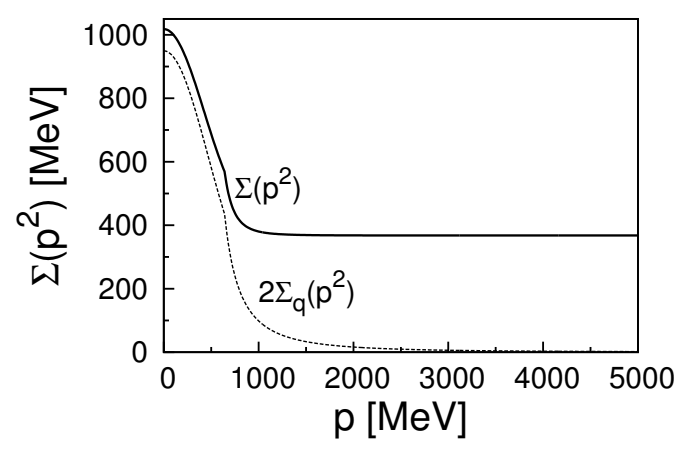

(a) $R=1 \mathrm{fm} \quad$ (i.e., $\Lambda=200 \mathrm{MeV}$ )

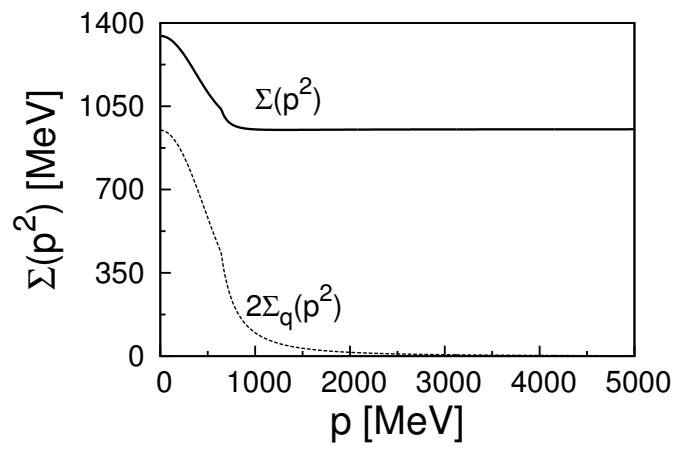

(b) $R \simeq 0.3 \mathrm{fm} \quad$ (i.e., $\Lambda=600 \mathrm{MeV}$ )

Figure 3: The scalar-diquark self-energy $\Sigma\left(p^{2}\right)$ as a function of the momentum in the running bare-mass case with [a] $R=1 \mathrm{fm}$ and [b] $R \simeq 0.3 \mathrm{fm}$. The diquark bare mass $m_{\phi}\left(p^{2}\right)=2 \Sigma_{q}\left(p^{2}\right)$ is also plotted with the dotted line for comparison.

\section{Dynamical Mass Generation without Chiral Symmetry}

Finally, we consider the zero bare-mass case of diquarks, $m_{\phi} \equiv 0$. The result is shown in Fig. (t) for the two cases of $R=1 \mathrm{fm}$ and $R \simeq 0.3 \mathrm{fm}$ on the diquark effective size. The self-energy $\Sigma\left(p_{E}^{2}\right)$ is always finite and takes a large value even for $m_{\phi} \equiv 0$. The mass generation mechanism in QCD is usually considered in the context of spontaneous chiral symmetry breaking. On the other hand, our scalar-diquark theory is composed by an effective scalar-diquark field $\phi(x)$ and does not have the chiral symmetry explicitly, although the original diquark is constructed by two quarks.

We consider that QCD has several dynamical mass-generation mechanism, even without chiral symmetry breaking. For example, while the charm quark has no chiral symmetry, some difference seems to appear between current and constituent masses for charm quarks: the current mass is $m_{c} \simeq 1.2 \mathrm{GeV}$ at the renormalization point $\mu=2 \mathrm{GeV}$ [四], and the constituent charm quark mass is $M_{c} \simeq 1.6 \mathrm{GeV}$ in the quark model. Furthermore the gluon is more drastic case. While the gluon mass is zero in perturbation QCD, the non-perturbative effect of the self-interaction of gluons seems to generate a large effective mass of $0.6 \mathrm{GeV}[\mathrm{D}]$, and the lowest glueball mass is about $1.6 \mathrm{GeV}$. Although the heavy quark and gluons do not have the chiral symmetry, they obtain large effective mass by non-perturbative effects. In this sense, the scalar diquark mass can be also generated by the gluonic effect. Thus, we consider that the mass generation mechanism is a general property of the strong interacting theory, and one typical mass generation is given by spontaneous chiral-symmetry breaking, which is also caused by the non-perturbative interaction. 


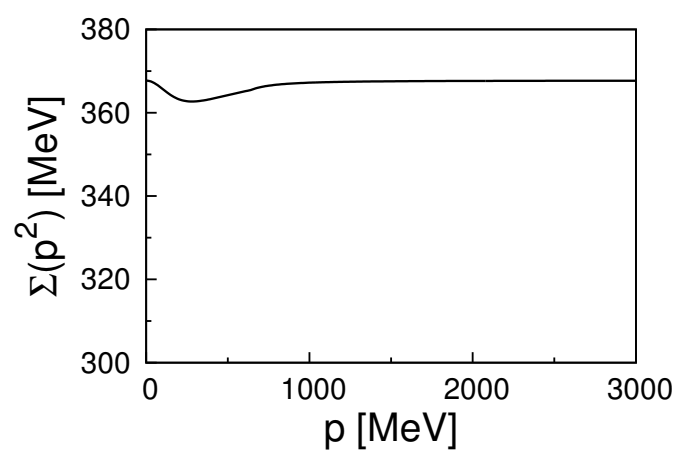

(a) $R=1 \mathrm{fm} \quad$ (i.e., $\Lambda=200 \mathrm{MeV}$ )

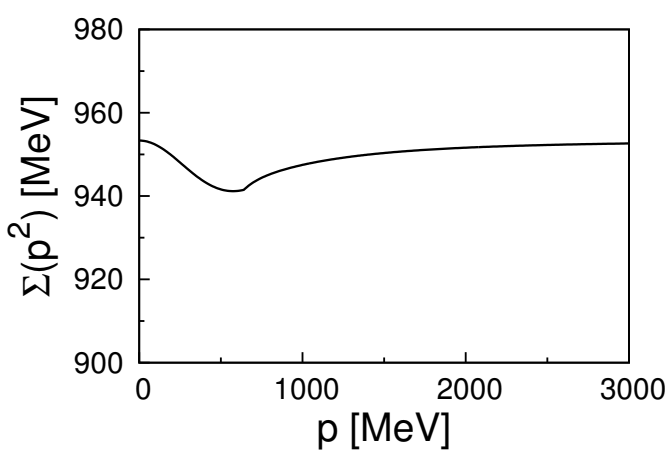

(b) $R \simeq 0.3 \mathrm{fm} \quad$ (i.e., $\Lambda=600 \mathrm{MeV}$ )

Figure 4: The scalar-diquark self-energy $\Sigma\left(p^{2}\right)$ as a function of the momentum in the massless case of $m_{\phi}=0$. The self-energy $\Sigma\left(p^{2}\right)$ is finite in both cases.

\section{Summary}

We have investigated the gluonic dressing effect to the scalar diquark, considering the size effect of the diquark in a hadron. The non-perturbative effect is evaluated in the Schwinger-Dyson (SD) formalism in the Landau gauge. Since the diquark is located inside a hadron, the diquark size $R$ must be smaller than the hadron $(\sim 1 \mathrm{fm})$ and larger than the constituent quark $(\sim 0.3 \mathrm{fm})$. We have considered two cases of the constant bare mass $m_{\phi}=600 \mathrm{MeV}$ and the running bare mass $m_{\phi}\left(p_{E}^{2}\right)=2 \Sigma_{q}\left(p_{E}^{2}\right)$. The diquark self-energy strongly depends on the size $R=\Lambda^{-1}$ in both cases, especially the small diquark ( $R \simeq 0.3 \mathrm{fm}$, i.e., $\Lambda=600 \mathrm{MeV}$ ) obtains a large effective mass by the gluonic dressing effect.

We find that the effective diquark mass is finite and large even for the zero bare-mass case, and the value strongly depends on the size $R$, which is an example of dynamical mass generation by the gluonic dressing effect, without chiral symmetry breaking.

\section{Acknowledgments}

This work is in part supported by the Grant for Scientific Research [Priority Areas "New Hadrons" (E01:21105006), (C) No.23540306] from the Ministry of Education, Culture, Science and Technology (MEXT) of Japan.

\section{References}

[1] J. Beringer et al. (Particle Data Group), Phys. Rev. D86 (2012) 010001 and update for the 2014 edition.

[2] K. Higashijima, Phys. Rev. D29 (1984) 1228.

[3] K.-I. Aoki, M. Bando, T. Kugo, M. G. Mitchard, and H. Nakatani, Prog. Theor. Phys. 84 (1990) 683.

[4] N. Yamanaka, T. M. Doi, S. Imai, and H. Suganuma, Phys. Rev. D88 (2013) 074036.

[5] S. Imai and H. Suganuma, arXiv:1401.7762 [hep-ph].

[6] H. Iida, H. Suganuma and T.T. Takahashi, Phys. Rev. D75 (2007) 114503.

[7] T. Iritani, H. Suganuma and H. Iida, Phys. Rev. D80 (2009) 114505. 